\title{
APLICACIÓN DEL MODELO DE NEGOCIO CANVAS E INFLUENCIA EN LA PRODUCTIVIDAD EMPRESARIAL DE LAS EMPRESAS OLIVÍCOLAS DEL DISTRITO LA YARADA LOS PALOS
}

\author{
THE APPLICATION OF THE CANVAS BUSINESS MODEL AND ITS INFLUENCE ON THE BUSINESS \\ PRODUCTIVITY OF OLIVE COMPANIES IN THE DISTRICT LA YARADA LOS PALOS.
}

Evelyn Massiel Portugal Mamani ${ }^{1}$

https://orcid.org/0000-0002-3113-9211 massiel.agroindustrial@gmail.com

Victor Samuel Damaso Márquez Tirado 2

https://orcid.org/0000-0001-5467-886X vsdmarquezt@gmail.com

\begin{abstract}
RESUMEN
La investigación tuvo como objetivo determinar la influencia del modelo de negocio Canvas en la productividad empresarial de las empresas olivícolas del distrito La Yarada Los Palos, en el año 2019. La metodología utilizado consistió en un diseño analítico correlacional, se trabajó con dos empresas olivícolas del sector denominados Sur Oliva S.A.C. y Baumann Crosby S.A. donde se evaluó el modelo de negocio Canvas desde dos perspectivas; lienzo Canvas y el cuestionario de 13 preguntas aplicado a 20 trabajadores y clientes, la productividad empresarial se evaluó con un cuestionario de 9 preguntas mediante la escala de Likert a 20 trabajadores y clientes de las unidades de estudio; los resultados de las empresas en estudio desde los nueve elementos del modelo Canvas fueron; Recursos clave, Actividades, Alianzas clave . También la Propuesta de valor. Además, el tipo de Segmento de mercado, relaciones con los clientes, Canales de distribución. Finalmente se estableció los costos (en la producción y procesamiento) y las fuentes de ingreso. El estadístico utilizado para la comprobación de la hipótesis fue el Chi cuadrado al $95 \%$ de confiabilidad donde fue significativo por lo que la productividad empresarial está influenciada por el modelo de negocio Canvas.
\end{abstract}

\footnotetext{
1 Universidad Privada de Tacna. Facultad de Ingeniería. Magíster en Administración y Dirección de Empresas

2 Universidad Privada de Tacna. Facultad de Ciencias Empresariales. Tacna, Perú.
} 
Palabras clave: Lienzo Canvas, productividad empresarial, modelo de negocio, sectorolivícola.

\begin{abstract}
The objective of the research was to determine the influence of the Canvas business model on the Business Productivity of the olive companies of the La Yarada Los Palos District, in 2019. The methodology used consisted of a correlational analytical design, we worked with two olive companies in the sector called Sur Oliva S.A.C. and Baumann Crosby S.A. Where the Canvas business model was evaluated from two perspectives; Canvas and the questionnaire of 13 questions applied to 20 workers and customers, business productivity was evaluated with a questionnaire of 9 questions using the Likert scale to 20 workers and customers of the units of study. The results of the companies under study from the nine elements of the Canvas model were; Key resources, Activities, Key alliances. Also the Value Proposition. In addition, the type of market segment, customer relationships, distribution channels. Finally, the costs (in production and processing) and the sources of income were established. The statistic used to test the hypothesis was the Chi-square at $95 \%$ reliability where it was significant so that business productivity is influenced by the Canvas business model.
\end{abstract}

Keywords: Canvas, business productivity, business model, olive sector.

\title{
INTRODUCCIÓN
}

En las empresas actuales urge la imperiosa necesidad de plantear un modelo de negocio apropiado ya que permitirá tener las características de los elementos que integran la empresa y las actividades que se desarrollan en ellas, además permite orientar el rumbo del negocio a fin de generar mayores utilidades, lo que es más importante garantizando la generación de valor en cada una de las tareas que se brinda en la atención de las necesidades y deseos de los clientes; motivo por lo cual la metodología Canvas, resulta una herramienta de suma importancia, ya que es completamente aplicable dentro de las pequeñas industrias de la ciudad especialmente en el sector olivícola por tratarse de un producto bandera de la región como es la aceituna.

Cabe resaltar que las empresas en cuestión no poseen un modelo de negocio, por ello no tiene información concreta de las capacidades de la empresa, sus potencialidades de producto, las necesidades de mercado y la presencia de la competencia, para establecer las estrategias adecuadas para mejorar la competitividad y productividad empresarial que permita optimizar la rentabilidad de las empresas en estudio. Por ello en el presente trabajo de investigación se tuvo como objetivo la aplicación del modelo de negocio Canvas y su influencia en la productividad empresarial del sector olivícola, en este caso se ejemplificó en las empresas empresas Sur Oliva S.A.C. y Baumann Crosby S.A., usando para ello una 
herramienta que en últimos años se está empleando en todo el mundo y en infinidad de negocios de distintas características sin embargo se puede aplicar a todos y cada uno de ellos.

Un modelo de negocio describe las bases sobre las que una empresa crea, proporciona y capta valor (Osterwalder, A., \& Pigneur, Y. , 2010). También el modelo de negocio es el mecanismo por el cual un negocio genera ingresos y beneficios y al mismo tiempo como una empresa sirve a sus clientes, el modelo de negocios aporta dos ideas cruciales; la primera es que debe generar teóricamente un beneficio mutuo tanto para el cliente y la empresa y la segunda es que debe ser explicado a través de unos pocos elementos clave (Caicedo, L, 2016).

Según Cáceres (2016) la metodología Canvas tiene un efecto medio con un puntaje de 2 a 3 en la escala valorativa, quedando así demostrado evidentemente en los resultados, que el efecto de la metodología Canvas en las Mypes de calzado es positivo. También Rivera (2016), este modelo de negocio ha sido validado con piscicultores y disminuye los niveles de riesgo e incertidumbre toda vez que el productor -que a su vez es proveedor de vísceras y cliente del alimento concentrado- ha estado involucrado en el diseño y despliegue del modelo. Asimismo Huchiyama (2016) propone un modelo de negocio de un restaurante móvil basado en el método Running Lean en la ciudad de Chiclayo, se determinaron los posibles riesgos del modelo de negocio, que existe una demanda insatisfecha, en cuanto a la calidad de los productos y servicios que brindan dichos establecimientos aledaños para satisfacer la necesidad culinaria de los clientes con menús con un alto valor nutricional, excelente combinación, presentación y con precios accesibles a todos ellos en un espacio único.

Rojas (2017) tuvo por finalidad proponer modelo de negocios para la Empresa Multisectorial de Ayash S.A. de Huaraz - 2017. De igual forma Cárdenas, Vargas \& Almanza (2017) el objetivo era adaptar el modelo de negocios Canvas, como una herramienta que contribuya en la competitividad de las Pymes en Lázaro Cárdenas, Michoacán. La misma trata de que las micro, pequeñas y medianas empresas, representan un papel importante en la economía de México, principalmente en la generación de empleos y su contribución en el producto interno bruto, por lo que es necesario que se busquen diferentes estrategias que contribuyan en su progreso, evitando su estancamiento y en muchos casos la desaparición.

Asimismo, Caicedo (2016) tiene como objetivo diseñar un modelo de negocios para la empresa Pulpa de Luruaco, utilizando el modelo Canvas como modelo referente con el fin de mantener una gestión administrativa y organizacional de la planta despulpadora de frutas que permita el fortalecimiento productivo. Basado en un análisis de los modelos de negocios aplicados y desarrollados, principalmente en el modelo Canvas; se modela y diseña un modelo de negocios aplicado a la agroindustria, en busca de un objetivo común como es el desarrollo social y económico de la región con la siembra, cosecha y procesamiento del mango criollo o mango de azúcar cosechado en las fincas o parcelas de las 50 familias que integran la Comunidad de Matamba.

Del mismo modo Choque (2018) tiene como objetivo proponer un plan de negocios basado en el modelo Canvas para determinar si es posible producir y comercializar derivados a base de Stevia en la ciudad de Arequipa. Para ello se elaboró un plan de negocios basado en el modelo Canvas para producir y comercializar derivados a base de Stevia en Arequipa, demostrando lo atractivo que puede ser para los inversionistas, ya que no solo las herramientas financieras son favorables para la ejecución del mismo, sino que está res paldado por una estrategia fuerte, un plan de marketing especifico y que responde a los gustos y preferencias del mercado, y un conjunto de planes: organización, financiero, etc.; que harían 
de este negocio una fuente de ingresos y de desarrollo, para el inversionista, los trabajadores y la comunidad. Finalmente, Casas \& Poveda (2017) tiene como objetivo diseñar el modelo de negocio para validar la idea de emprendimiento "Verduritas" en la ciudad de Bogotá. Mediante el uso de la herramienta Canvas se obtiene un recurso no solo estratégico sino potencial con el que los emprendedores pueden desarrollar sus ideas y transformarlas en empresas exitosas.

Las micro, pequeñas y medianas empresas (Pymes), son consideradas como agentes económicos, ya que considerable parte de la economía y la población dependen de su actividad y desempeño. En países que conforman la Unión Europea, América Latina y el Caribe las pymes, conforman el $99 \%$ del total de empresas, son una considerable fuente de empleo (67\%), teniendo participación en diversos ámbitos de la producción y servicios. Las Pymes tienen un mayor peso como generadores de empleo que como centros de producción, los niveles de productividad son menores al de grandes empresas (Néstor, 2015, p.29).

En países en vías de desarrollo como el Perú, la economía está sostenida por las Micro, Pequeñas y Medianas Empresas (MYPE). De acuerdo a información proporcionada por la SUNAT; al año se registran unas 300,000 empresas, pero se cierran unas 200,000; teniendo como motivos del cierre la falta de segmentación y planificación, el desconocimiento del negocio, y del mercado, lo que genera insostenibilidad en el tiempo, los ingresos no acordes con sus expectativas y que basen su desarrollo empresarial en el empirismo, anotó la Cámara de Comercio de Lima en el año 2012 (Mejía, 2003). Asimismo, el Perú es uno de los principales productores y exportadores mundiales de aceituna negra entera de maduración y fermentación natural, donde en las regiones (Tacna, Arequipa, Ica y Moquegua) existen aproximadamente unos 3 mil aceituneros, los cuales producen en promedio unas 25000 toneladas de aceituna entera por año. El $5 \%$ de la producción peruana de olivo se dedica a aceite y el $95 \%$ se procesa en forma de aceituna entera.

De acuerdo a la Oficina Comercial de Exportación Regional, menciona que Tacna cuenta con 2500 Mypes principalmente de los sectores agro, como olivo, orégano, páprika, cebolla, entre otros. (citado en Delgado, 2018). Sin embargo, en las organizaciones actuales urge la necesidad de plantear un modelo de negocio apropiado ya que permitirá tener las características de los elementos que integran la empresa y las actividades que se desarrollan en ellas, además permite orientar el rumbo del negocio a fin de generar mayores utilidades, lo que es más importante garantizando la generación de valor en cada una de las tareas que se brinda en la atención de las necesidades y deseos de los clientes; motivo por lo cual la metodología Canvas, resulta una herramienta de suma importancia, ya que es completamente aplicable dentro de las pequeñas indus trias de la ciudad.

Para la investigación se considera a las empresas Agroindustrias Sur Oliva S.A.C. y Bauman \& Crosby S.A.C. ubicadas en el distrito de la Yarada- los Palos, que vienen operando en el sector agroindustrial del olivo, a pesar de su presencia en el mercado por largo periodo (la primera de la mencionada 5 años en el sector y la segunda más de 25 años en el rubro), aún no logra posicionarse, debido a que no tiene bien determinado el segmento de mercado, los canales que emplea para llegar a los clientes no son tan eficientes y al momento de desarrollar las actividades no generan valor para satisfacer las necesidades de los clientes y permitir un reconocimiento hacia la empresa. Las empresas en cuestión no poseen un modelo de negocio, por ello no tiene información concreta de las capacidades de la empresa, sus potencialidades de producto, las necesidades de mercado y la presencia de la competencia, para establecer las estrategias adecuadas para mejorar la competitividad y productividad 
empresarial que permita mejorar la rentabilidad de las empresas en estudio.

Respecto a lo antes mencionado, en las empresas olivícolas es que se implementará el modelo de negocios Canvas que proporcionará información acerca de los recursos y las actividades que se realizarán en ellas, que permitirá optimizar la productividad empresarial y ésta permitirá alcanzar los objetivos de la empresa tomando en cuenta la relación de los recursos invertidos y los resultados de los mismos, este aspecto es importante para una buena gestión empresarial y viabilidad del mismo. Asimismo, se logrará un efectivo control sobre las metas y objetivos, el fomento de sinergias, la creatividad, innovación tecnológica, delegación de responsabilidades, planificación de tiempos, potenciación de la conciliación y flexibilidad laboral que son aspectos clave para lograr la productividad empresarial de la empresa y por ende ser competitivo en el sector.

\section{OBJETIVOS}

Determinar la influencia del modelo de negocio Canvas en la productividad empresarial de las empresas olivícolas del distrito La Yarada Los Palos, en el año 2019.

\section{METODOLOGÍA}

La elaboración del Lienzo Canvas, incluyó los 9 bloques que lo constituyen, los mismo son (Segmentación del cliente, Propuesta de valor, Canales de distribución, Relación con el cliente, Flujo de ingresos, Recursos Clave, Actividades claves, Socios claves y Estructura de costos). - Productividad empresarial: Las encuestas que se elaboraron abarcan lo siguientes aspectos (Fomento de Sinergias, Incentivo de la creatividad, Innovación tecnológica, Planificación de actividades, Delegación de Funciones, Flexibilidad Laboral, Medición de operaciones y procesos, eficacia laboral general y Objetivos y metas).

El tipo de investigación es aplicada. El diseño de investigación corresponde al descriptivo correlacional, estas asociaciones nos dan pistas para suponer influencias y relaciones causa-efecto (Vara Horna, 2010). El nivel de investigación corresponde a un estudio de Nivel Activa o Aplicada. Ámbito y tiempo social de la investigación es el Distrito La Yarada-Los Palos Km 25.

La población son las empresas olivícolas del dicho distrito. La muestra fueron dos empresas del rubro olivícola: Sur Oliva S.A.C y Baumann Crosby S.A. El tipo de muestreo empleado fue el no probabilístico de selección intencional típico y necesario cuando se realizan investigaciones cualitativas, documentales.

Para el Modelo de Negocio Canvas, se elaboró el Lienzo de Negocio Canvas además del cuestionario de 13 preguntas que se aplicó a 20 personas entre clientes y trabajadores de las empresas en estudio. Para la Productividad empresarial, se aplicó un cuestionario de 9 preguntas mediante la escala de Likert a 20 trabajadores y clientes porque se requiere analizar y valorar aspectos de objetivos y metas de la empresa, fomento de innovación, tecnologías de proceso, recurso humano que permita analizar y evaluar la variable dependiente para su corroboración.

El instrumento utilizado para evaluar la variable independiente se consideró al empleado en la investigación denominado Modelo Canvas: 


\section{Tabla 1}

\section{Resultado de campo del análisis de bloques del modelo Canvas en las empresas}

\begin{tabular}{|c|c|}
\hline $\begin{array}{l}\text { Bloques del } \\
\text { modelo Canvas }\end{array}$ & Empresa Sur Oliva SAC \\
\hline $\begin{array}{l}\text { Asociaciones } \\
\text { clave }\end{array}$ & $\begin{array}{l}\text {-Alianzas con prove edores } \\
\text {-Prove dores de e nvases: } \\
\text {-Provee dores de maquinarias y equipos } \\
\text {-Provee dores de combustible } \\
\text {-Proveedores de otros materiales } \\
\text { - Bancos y seguros }\end{array}$ \\
\hline $\begin{array}{l}\text { Actividades } \\
\text { clave }\end{array}$ & $\begin{array}{l}\text {-Producción } \\
\text {-Actividades administrativas, Comercio } \\
\text { Exteriory Logís tica }\end{array}$ \\
\hline $\begin{array}{l}\text { Propuesta de } \\
\text { valor }\end{array}$ & $\begin{array}{l}\text { - La empresa Sur Oliva S.A.C, es una } \\
\text { empresata cneña, que busca consolidarse } \\
\text { y ser líder en el merca do peruano; } \\
\text { ofreciendo productos al mejor precio. }\end{array}$ \\
\hline
\end{tabular}

\section{Empresa Baumann \& Crosby SA}

-Alianza con proveedores (proveedores de insumos,
envases, servicios, maquinarias y equipos, otros materiales).

-Alianza con asociaciones (Proolivo) y empresas procesadoras de aceite de oliva y de rivados.

- Bancos ys eguros

Procesamiento de Aceituna negra (Recolección,

Trans porte, Pre paración de Salmuera, Fermentación,

Selección y clasificación, Envasado y Muestreo)

Procesamiento de Aceituna verde (Recolección, tra ns porte, preparación de soda cá ustica, tra tamiento con lejía, lavados, salmuera, fermentación, selección y clasificación, adición de salmuera, envasado).

- Va riada presentación de aceitunas, como aceituna negra natural en salmuera: Variedades Criolla, Ascolana, Empeltre y Pendolino y Aceituna verde aderezada en sa Imuera, va riedades criolla y As colana. Aceitunas negras o verdes deshuesadas, verde o negra en rodajas y verde rellena con rocoto o pimiento.

- Agra da ble y diferenciado sabor

\begin{tabular}{|c|c|c|}
\hline $\begin{array}{l}\text { Relaciones con } \\
\text { clientes }\end{array}$ & $\begin{array}{l}\text { Comunicación Indirecta (mediante } \\
\text { bróker) con los clientes intemacionales, } \\
\text { pues la empresa exporta sus productos a } \\
\text { su principal comprador: Brasil. Cuenta } \\
\text { con compradores nacionales, } \\
\text { brindándoles descuentos a los mayoristas }\end{array}$ & $\begin{array}{l}\text {-Encuesta mediante formato de atención de quejas de } \\
\text { los clientes. } \\
\text {-Des pachos de contenedores del producto en lapso de } \\
\text { tiempo acordado. }\end{array}$ \\
\hline Canales & $\begin{array}{l}\text { Indirecto: venta a empresas } \\
\text { exporta doras brasileras, luego venderán } \\
\text { a clientes finales. La a ceituna no } \\
\text { exporta da es vendida al mercado local: } \\
\text { Mayorista-detallista-consumid or final. }\end{array}$ & $\begin{array}{l}\text { Indirecto: venta a empresas exportadoras brasileras, } \\
\text { luego venderán a clientes finales. La aceituna no } \\
\text { exportada es vendida al mercado local: Mayorista- } \\
\text { detallista-consumidorfinal. }\end{array}$ \\
\hline Recurs os clave & $\begin{array}{l}\text {-Ma quinaria, equipo, materiales. } \\
\text {-Ma te ria prima e insumos. } \\
\text {-Terre no e infraestructura } \\
\text {-Pers onal especializado. }\end{array}$ & $\begin{array}{l}\text { Ma quinaria y equipos (campo y proce samiento de } \\
\text { a ceituna) } \\
\text {-Ma te ria prima e insumos de campo y procesamiento } \\
\text {-Ma te riales de laboratorio } \\
\text { - Infra estructura de campo y procesamiento. } \\
\text {-Personal profesional y ma no de obra. } \\
\text {-Vehículos }\end{array}$ \\
\hline $\begin{array}{l}\text { Segmentos de } \\
\text { mercado }\end{array}$ & $\begin{array}{l}\text {-Import Foods Ltda, } \\
\text { - Alimentos Zaeli } \\
\text { - Rofimex } \\
\text { - Sandeleh Alimentos Ltda } \\
\text { - Frugal Importadora } \\
\text { - Comercial Importadora La ctinos } \\
\text { Napolitanos } \\
\text { - Komport Comercial e Importadora S.A. }\end{array}$ & $\begin{array}{l}\text {-Merca do internacional como: Chile, Brasil e Italia. } \\
\text { - Merca do nacional ylocal, teniendo a las sgts. Empresas } \\
\text { como clientes: Aceta ex S.A.C; Aeromater S.A; } \\
\text { Agroindustrias Calport E.I.R.L; Agroindustrias del Sur S.A; } \\
\text { Agroindustrias Gonzales E.I.R.L; Alimentos Finos Rila Chile } \\
\text { Ltda; Antonella Gianelli Calderon; Brigida Chura Calizaya; } \\
\text { Deinal S.A.C; Edith Rodriguez Giraldes; Eloy Cutipa } \\
\text { Huanca; Exporta ciones Mirsa E.I.R.L; Fundo La Noria; } \\
\text { Gustavo Ayca Tintaya; Jose Velásquez Quispe; La } \\
\text { Genovesa AgroindustriasyProensa }\end{array}$ \\
\hline $\begin{array}{l}\text { Costos } \\
\text { Producción }\end{array}$ & $\begin{array}{l}\text { Costos de procesamiento de aceituna } \\
\text { verde y Negra (Ma teria Prima, insumos, } \\
\text { servicios básicos y mano de obra) }\end{array}$ & $\begin{array}{l}\text { Costos, Producción (Ma te ria prima, Ma no de obra y } \\
\text { Gas tos indirectos) y Procesamiento (Ma teria Pri ma, } \\
\text { Insumos, Ma no de Obra del personal profesional y } \\
\text { obreros, Servicios Básicos y Ga stos por condición de } \\
\text { trabajo) }\end{array}$ \\
\hline $\begin{array}{l}\text { Fuente de } \\
\text { Ingresos }\end{array}$ & $\begin{array}{l}\text { Venta de aceituna negra } \\
\text { Venta de aceituna verde }\end{array}$ & $\begin{array}{l}\text { Venta de aceituna negra de diversas variedades } \\
\text { Venta de aaceituna verde de diferentes va riedades. }\end{array}$ \\
\hline
\end{tabular}




\section{RESULTADO}

\section{Modelo de negocio Canvas}

En la Figura 1 se denota que el 65\% de los encuestados son del sexo femenino, mientras que el $35 \%$ son del sexo masculino. En la Figura 2; se observa que el $40 \%$ de los encuestados tiene más de 40 años, seguido de un $25 \%$ que tiene entre 31 y 40 años, al igual que el $25 \%$ con edades entre 20 y 30 años, finalmente la edad del $10 \%$ oscila entre 19 años en adelante.
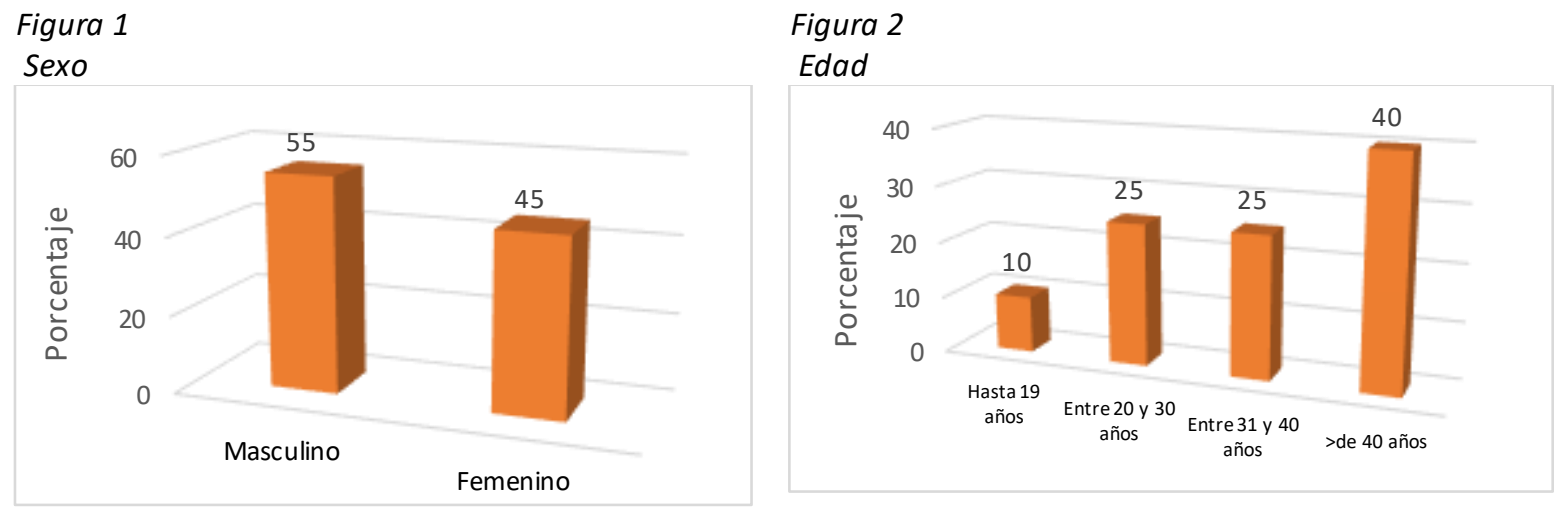

Respecto a si probó algunos de los tipos de aceituna que se procesan en la empresa, el $65 \%$ de los encuestados, manifestó que si lo hicieron, ello para sentir el sabor y ver lo agradable puede ser para el público, mientras que el $35 \%$ dice que no lo hicieron. En la cuarta pregunta referentes a quienes consumen o consumirían la aceituna negra, el 75\% manifestó que sería el público en general, el $15 \%$ dijo que serían algunos adultos, mientras que el $10 \%$ afirmó que los consumidores serían los compradores.

Respecto (figura 5) a quienes consumen o consumirían aceituna verde, el $60 \%$ de los encuestados manifestaron que los harían los compradores, el $25 \%$ afirmo que lo harían algunos adultos y el $15 \%$ que serían el público en general. Se denota que el $75 \%$ de los encuestados considera que la calidad es un valor relacionado con la aceituna que se produce la empresa, el $15 \%$ indica que los precios buenos son valores relacionados a las aceitunas que se produce en la empresa, así mismo el $10 \%$ considera que la confianza es el valor más estrechamente relacionado con la aceituna que se produce en la empresa.

Figura 3 Probó algún tipos de aceituna que procesan las empresas Baumann Crosby S.A. y Sur Oliva.

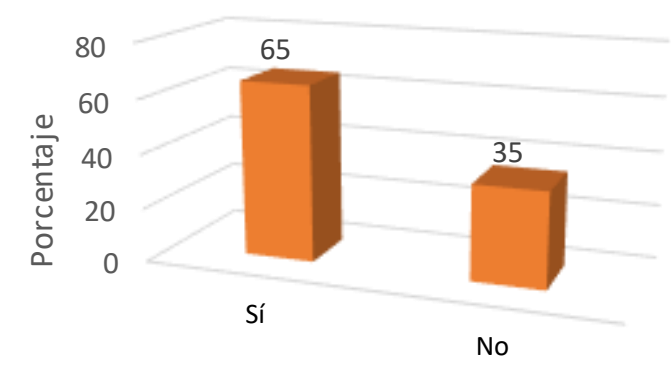

Figura 4 ¿Quienes consumen o consumirían la aceituna negra?

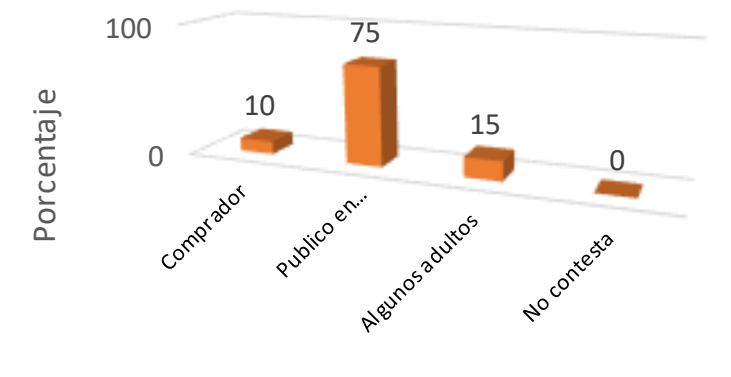


Figura 5 ¿Quienes consumen o consumirían la aceituna verde?

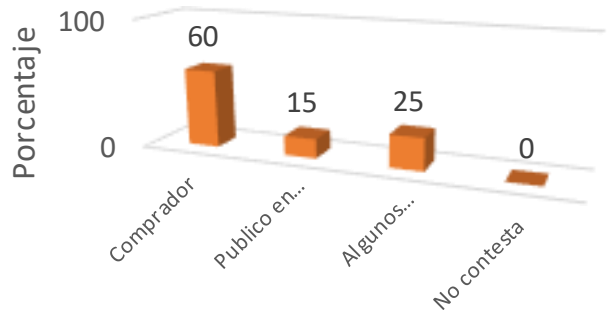

Figura 7 ¿Tiene conocimiento de las características de exquisitez de la aceituna negra en relación a la aceituna de la competencia?

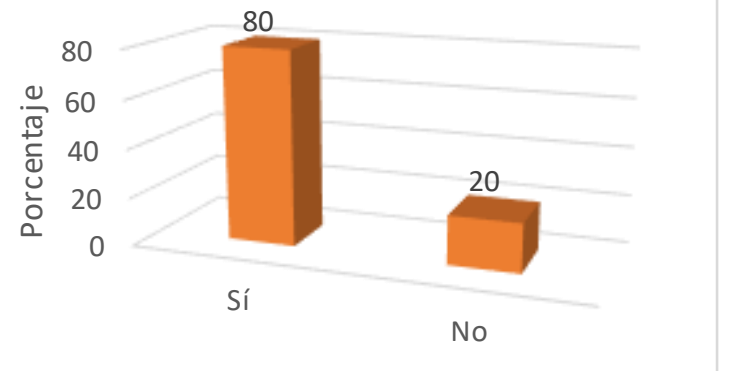

Figura 9

¿Cómo le gustaría que fuera el envase?

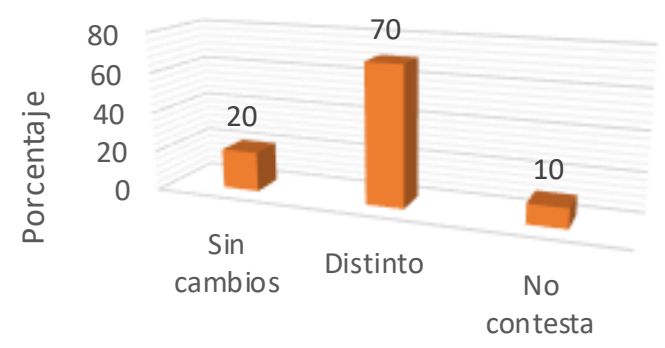

Figura 11 Cuánto más estaría dispuesto a pagar el kg de aceituna conociendo sus ventajas comparativas respecto a la competencia.

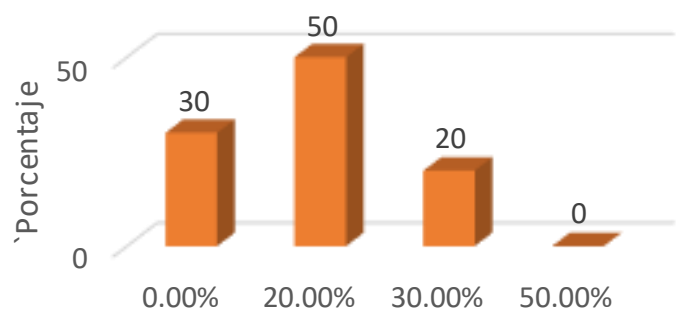

Figura 6 ¿Qué valores relaciona con la aceituna que produce la empresa Baumann Crosby?

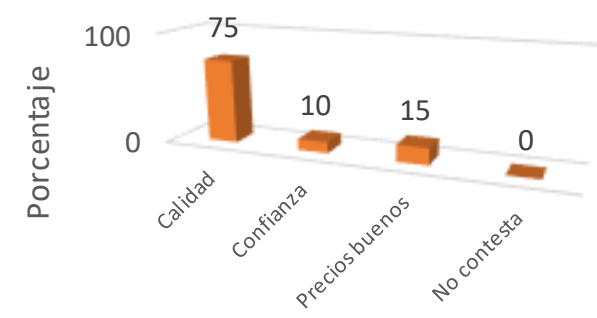

Figura 8 ¿Considera que las ventajas que posee lo podrían incentivar a comprar este tipo de aceituna con más frecuencia?

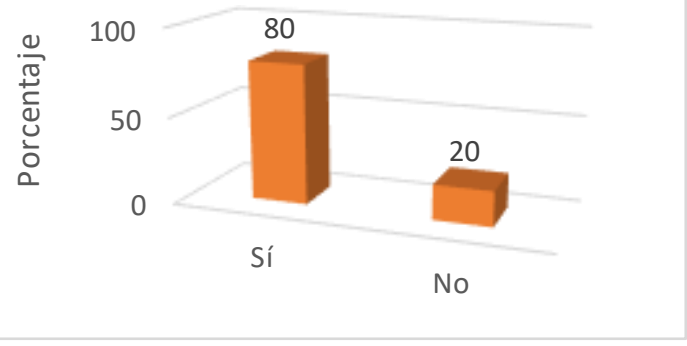

Figura 10 ¿Qué atributos destaca como importantes en el momento de la decisión de compra?

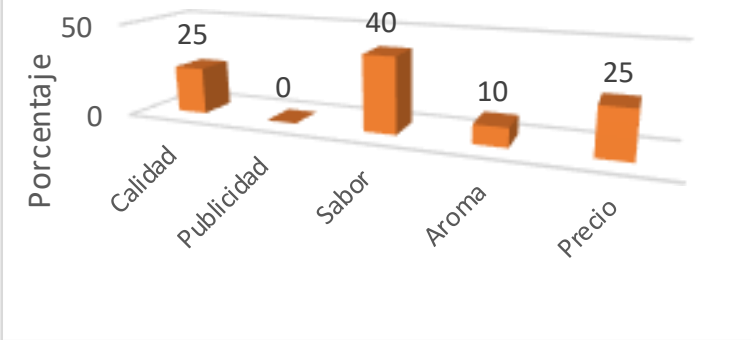

Figura 12 ¿Cuáles son las alianzas estratégicas que establece la empresa?

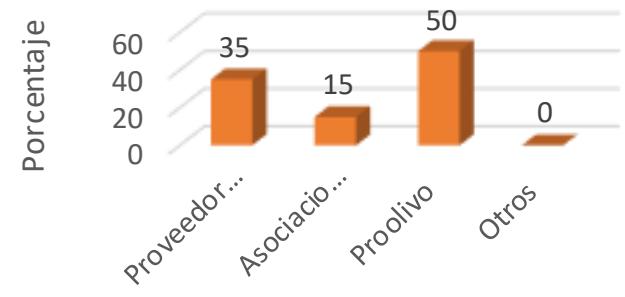

Figura13 ¿Conoce los puntos de venta de la empresa?

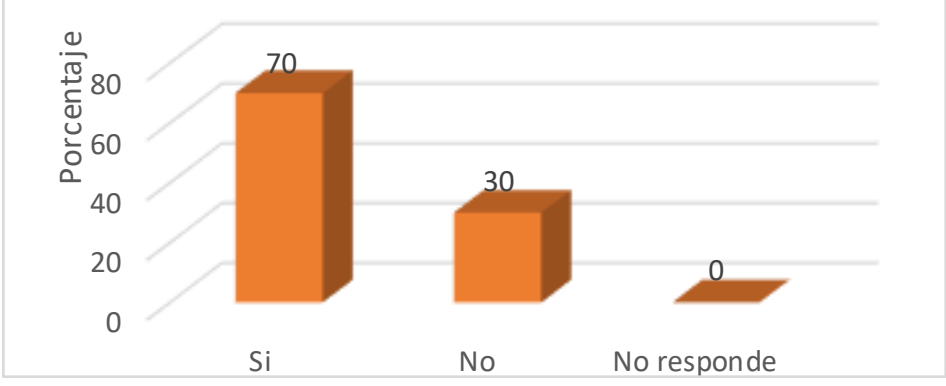


En la Figura 7, se hace referencia a las características de exquisitez de la aceituna negra en relación a la competencia, en la misma se ve que el $80 \%$ de los encuestados señalan que sí conocen el sabor de la aceituna que se procesa en la empresa, mientras que el $20 \%$ dijo no diferenciar el sabor característico de la aceituna elaborada en la empresa.

Se observa que el $80 \%$ de los encuestados indican que conocen las ventajas que posee la empresa podrían incentivar a comprar la aceituna con más frecuencia, mientras que el $20 \%$ considera que no es una ventaja que incentivaría a comprar la aceituna.

De acuerdo a la figura 9 , se denota que el $70 \%$ de los encuestados manifiesta el desea de cambiar el envase, el $20 \%$ señala no desea alguna variación y el $10 \%$ no responde a la pregunta.

El $40 \%$ de los encuestados considera que el sabor es un atributo destacado como importante en el momento de la decisión de compra, mientras que para el $25 \%$ de los encuestados la calidad y el Precio son atributos destacados, finalmente el $10 \%$ manifiesta que el aroma será el factor decisivo en la decisión de compra, puesto que un aroma a zapatería demostraría un producto en mal estado. En la figura 11, se observa que el $50 \%$ de los encuestados, estarían dis puestos a pagar un $20 \%$ por mas, el $30 \%$ manifiesta que no pagaría un $\%$ más sobre el precio de los productos y el $20 \%$ dice que pagaría $30 \%$ más. En la Figura 12 se observa que el $50 \%$ de los encuestados manifiestan que ProOlivo forma parte de la alianza estratégica que tienen las empresas mencionadas en el presente trabajo, el $35 \%$ manifiesta que con los proveedores se entablan alianzas estratégicas, finalmente el $15 \%$ define a las Asociaciones olivícolas como fuentes para establecer Asociaciones olivícolas.

De acuerdo a los datos observados en la figura 34 , se observa que el $70 \%$ de los encuestados conoce los puntos de venta de las empresas olivícolas, asi mismo el 30\% manifiesta lo contrario, debido a que estos últimos compran a intermediarios.

\section{Productividad empresarial}

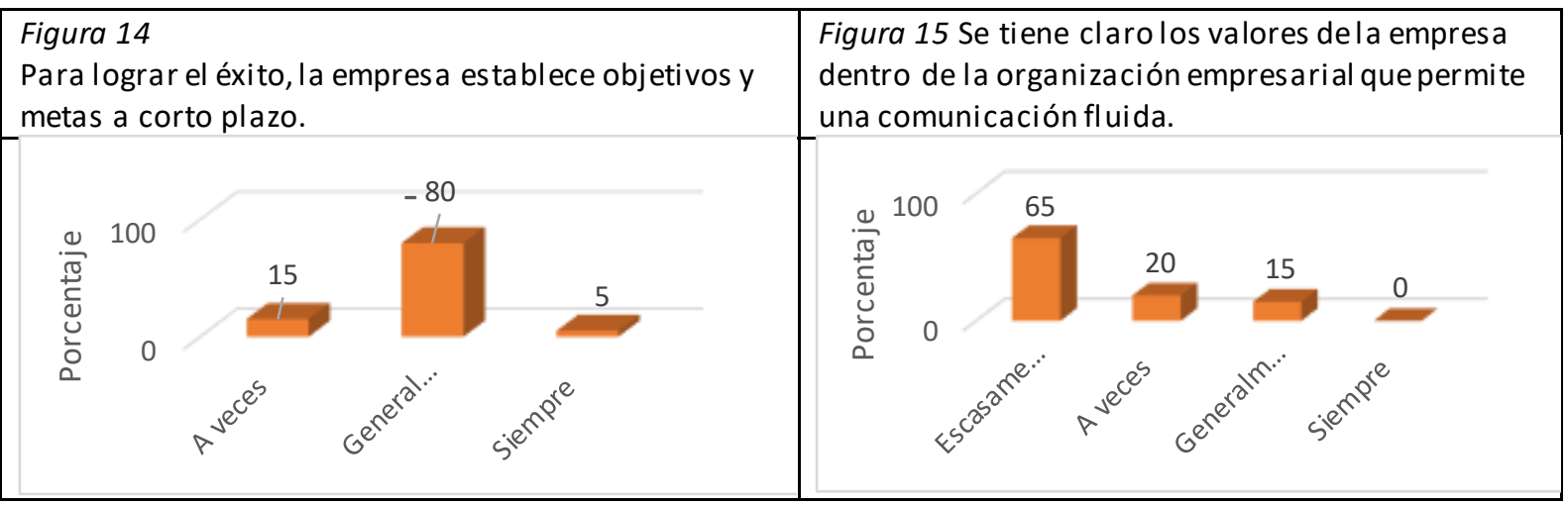




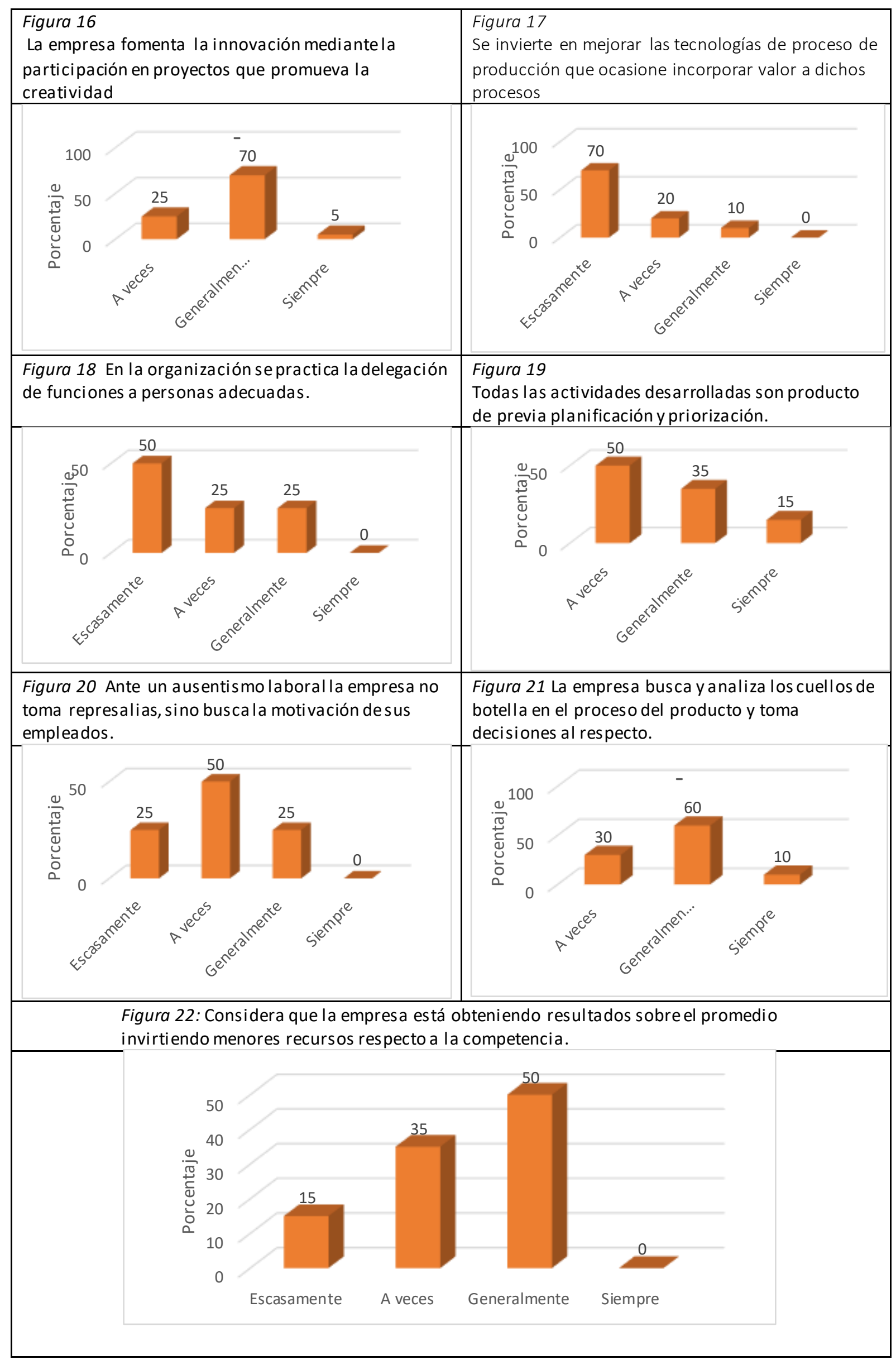


Se observa que el $80 \%$ de los encuestados manifiesta que las empresas olivícolas Generalmente establecen objetivos y metas a corto plazo; el $15 \%$ dice que a veces se establecen objetivos y metas a corto plazo y finalmente el $5 \%$ afirma que siempre se establecen objetivos y metas a corto plazo. En la figura 15, el $65 \%$ manifiesta que escasamente se tienen presentes los valores de empresa, el $20 \%$ dice que a veces los valores de la empresa son claros, por lo que no se mantiene una comunicación fluida y el $15 \%$ señala que generalmente. En la figura 16, se observa que el $70 \%$ de los encuestados señala que generalmente las empresas fomentan la innovación participando en proyectos que promueven la creatividad; asi mismo el $25 \%$ manifiesta que a veces se promueve la participación en proyectos para promover la creatividad y el $5 \%$ manifiesta que siempre promueven la innovación mediante la participación en proyectos. En la figura 17, el $70 \%$ de los encuestados afirma que escasamente se invierte en mejorar las tecnologías de proceso de producción, el $20 \%$ manifiesta que a veces las empresas promueven las mejoras de las tecnologías de proceso de producción y el $10 \%$ dice que generalmente. Se observa que del $100 \%$ de los encuestados, el $50 \%$ manifiesta que escasamente en las organizaciones se delegan funciones, mientras que el $25 \%$ indica que a veces se delegan funciones y el otro $25 \%$ señala que generalmente se delegan funciones. En la figura 19 , se observa que el $50 \%$ de los encuestados considera que a veces las actividades son productos de previa planificación, el $35 \%$ señala que generalmente se planifican las actividades y el $15 \%$ indica que siempre se planifica las actividades a realizarse. Se denota que el $50 \%$ de las personas considera que las empresas a veces no toman represalias ante el ausentismo laboral, el restante $25 \%$ indica que escasamente toman represalias y el $25 \%$ indica que generalmente lo hacen.

En la figura 21 se puede observar que el $60 \%$ de los encuestados generalmente las empresas buscan y analizan los cuellos de botella en proceso del producto, el $30 \%$ señala que a veces y el $10 \%$ indica que siempre. Se denota que el $50 \%$ de los encuestados generalmente considera que las empresas están obteniendo resultado invirtiendo menos recursos comparados con la competencia, mientras que el $35 \%$ señala que a veces se obtiene resultados favorables y el $15 \%$ indica que escasamente esto ocurre.

El $80 \%$ considera que las ventajas que posee el producto podría incentivar a comprar la aceituna con más frecuencia; asimismo el $70 \%$ menciona que le gustaría que el envase fuera distinto; el 80\% identifica como atributo importante al sabor al momento de decidir la compra; el $70 \%$ de los mencionados señala que si conoce los puntos de venta de la empresa; así como el $50 \%$ indica que existe una alianza estratégica con ProOlivo.

Respecto a la productividad; El $80 \%$ de los encuestados menciona que la empresa establece objetivos y metas a corto plazo; el $65 \%$ señala que escasamente se tiene claro los valores de la empresa; el $70 \%$ indica que la empresa fomenta la innovación; el $10 \%$ sostiene que generalmente se invierte en mejorar las tecnologías de proceso de producción; el $60 \%$ menciona que generalmente la empresa busca y analiza los cuellos de botella en el proceso del producto.

Al análisis del $X^{2}$ con un nivel de significancia del $5 \%$ y 1 grado de libertad, se obtuvo un valor de $X^{2}$ (cal) igual a 6.55 La comparación de los valores encontrados muestra significancia. Por lo que la productividad empresarial está influenciando a los elementos del modelo Canvas en el sector olivícola del distrito La Yarada-Los Palos de la provincia de Tacna.

Considerando que en la mayoría de los casos, sino en todas las empresas olivícolas de la zona de La Yarada-Los Palos se gestionan de manera empírica o en el mejor de los casos en base a principios básicos de un negocio que busca satisfacer al cliente y mantener la calidad 
de sus productos; este trabajo propone la utilización de una herramienta de negocios como es Canvas Business Model para revisar el concepto mismo del negocio y entregar objetivos claros para poder completar la consolidación de la empresa que se encuentra en un nivel alto, en calidad y servicio para que esta empresa pueda encontrar nuevas y creativas estrategias que lo lleven a posicionarse entre los más destacados ya no a nivel regional sino nacional o internacional.

\section{CONCLUSIONES}

El modelo de negocio Canvas influyó en la productividad empresarial en el sector olivícola del distrito La Yarada-Los Palos de la provincia de Tacna, porque el estadístico chi cuadrado muestra significancia al $5 \%$ de probabilidad. En consecuencia la hipótesis general queda aceptada.

Los recursos, actividades y alianzas clave influyen en la productividad empresarial de las empresas olivícolas del distrito La Yarada Los Palos, ya que el $75 \%$ de los encuestados considera que la calidad es un valor relacionado con la aceituna que produce la empresa. Asimismo, el $50 \%$ de los encuestados afirman que las actividades son previamente planificadas e identifica a la alianza estratégica establecida con Proolivo en un $50 \%$ según los encuestados.

La propuesta de valor ejerce un impacto en la productividad empresarial de las empresas olivícolas del distrito La Yara Los Palos, el mismo se corrobora ya que el $80 \%$ de los encuestados mencionan que tienen conocimiento de las características de exquisitez de la aceituna negra respecto a la competencia, así como el $80 \%$ de los encuestados refieren que las ventajas del producto lo pueden incentivar a comprar con mayor frecuencia y por ende mejorar sus ingresos y productividad empresarial.

El tipo de Segmento de clientes, relaciones con los clientes y canales de distribución contribuyen en la productividad empresarial de las empresas olivícolas del distrito La Yarada Los Palos, el mismo es corroborado ya que el $75 \%$ de los encuestados menciona que es el público general es el que consume la aceituna negra y el $60 \%$ menciona que quienes consumen la aceituna verde son los compradores respectivamente.

La estructura de costes y las fuentes de ingreso influyen en la productividad empresarial de las empresas olivícolas del distrito La Yarada Los Palos, ello se ratifica porque el $50 \%$ de los encuestados generalmente considera que las empresas están obteniendo resultados sobre el promedio invirtiendo menores recursos respecto a la competencia.

\section{RECOMENDACIONES}

Se sugiere la aplicación de un modelo de negocio basado en la innovación que puede adaptarse y ser exitoso por la calidad de su aplicación que promueva el apoyo de los empresarios para ser competitivos y mejorar la productividad empresarial.

Se sugiere la implementación de sistemas de gestión de calidad que permita mejorar las líneas de producción de las empresas olivícolas especialmente en cuanto a infraestructura, tecnologías de procesamiento y participación en asociaciones que permita mejorar la productividad empresarial.

Se recomienda difundir las ventajas del producto que le da el valor añadido respecto a la competencia y la tecnología para construir un portafolio de productos que permita satisfacer las necesidades del mercado del sector olivícola mejorando la rentabilidad del mismo apoyando así el desarrollo de la economía regional por medio de la agroindustria.

Se recomienda establecer políticas empresariales de comunicación basadas en la calidad, sabor, marca, precio, resaltando las ventajas comparativas del producto en estudio a partir de una encuesta aplicada lo cual es fundamental para el desarrollo exitoso de una 
startup, especialmente para establecer relaciones con los clientes.

Se sugiere asignar los recursos financieros que permita el proceso de innovación en cada bloque del modelo Canvas para que se realice con holgura económica y sin contratiempos, con una consideración diferencial de sus costos y un tratamiento específico.

\section{REFERENCIAS BIBLIOGRÁFICAS}

Andrade, S. (2012). Metodología Canvas, una forma de agregar valor a sus ideas de negocios. Obtenido de Innovacion.cl:

http://www.innovacion.cl/reportaje/metodologia-canvas-la-nueva-forma-deagregar-valor/

Buendía Rice, E. A. (2013). El papel de la Ventaja Competitiva en el desarrollo económico de los países. Revista Análisis Económico,, pp.155-178.

Caballero, R. (2013). Niveles de investigación. Investigación.

Cáceres, C. (2016). Efecto de la metodología Canvas para la innovación en modelos de negocio de la Mype formal de calzado de Trujillo, año 2106. Trujillo.

Caicedo, L. (2016). Diseño de un modelo de negocio para la gestión productiva de una planta procesadora de pulpa de mango de la universidad del norte. Barranquilla.

Cárdenas, R. vargas, J. \& Almanza, R. (2017). Adaptación del modelo Canvas como herramienta competitiva para las pymes de Lázaro Cárdenas Michoacán. México.

Carro, R. \& Gonzales, D. (2005). Productividad y Competitividad. Administracion de la Operaciones, 18.

Casas, R; Vargas, J. \& Almanza, R. (2017). Adaptación del modelo Canvas como herramienta competitiva para las mpymes de Lázaro Cárdenas, Michoacán. Revista Pymes, Innovación y Desarrollo, 45-65.

Casas, W. \& Poveda, J. (2017). Modelo de negocio para validad el emprendimiento "verduritas" en la ciudad de Bogotá. Bogotá: Universidad Libre de Bogotá.

Chirinos, C. R. (2011). Nicho de mercado: El enfoque desde el océano azul. Ingeniería Industrial $n^{\circ} 29$, pp. 171-181. Obtenido de Universidad de Lima: http://revistas.ulima.edu.pe/index.php/Ingenieria_industrial/article/view/233/209

Choque, T. (2018). Plan de negocioos basado enn el modelo Canvas para la factibilidad de la producción y comercialización de derivados a base de Stevia en Arequipa. Arequipa.

Cornejo, M. y. (2017). Modelo de negocio para una cafeteria organica en la ciudad de Chiclayo. Chiclayo: Universidad Catolica Santo Toribio de Mogrovejo.

Corvo, T. (2018). Lifeder.com. Obtenido de Productividad Empresarial: Tipos, Factores, Importancia: https://www.lifeder.com/productividad-empresarial/ 
Delgado, L. M. (2016). Propuesta de Modelo Asociativo para la Internacionalizacion de las Pequeñas y Medianas empresas Agroindustriales de aceituna de la region Tacna. Veritas Et Scientia, pp. 992-1001.

Espinosa, R. (2013). Segmentación de mercado, concepto y enfoque. Obtenido de https://robertoespinosa.es/2013/09/17/segmentacion-de-mercado-concepto-yenfoque/

Expertemprende. (2017). Guía didáctica del Modelo Canvas. Obtenido de http://culturaemprendedora.extremaduraempresarial.es/wpcontent/uploads/2012/09/Guia-Did\%C3\%A1ctica_Modelo-Canvas.pdf

Garcia, Y. (2000). Estudio de pre factibilidad para la instalación de una planta procesadora de aceite de oliva virgen. . Lima: Universidad Agraria La Molina.

Garrido, M. (1991). Estudio de las características físico químicas de la aceituna estilo sevillana. Yauca- Arequipa. Arequipa.

Garzozi Pincay, R. M. (2014). Planes de Negocios para Emprendedores. Union Europea: Iniciativa Latinoamericana de Libros de Texto Abiertos.

Hill, Charlie \& Jones, Garet. (2009). Administracion estratégica.8va.ED. Mexico: Interamericana Editores, S.A. de C.V.

Huchiyama, M. y. (2016). Diseño y propuesta de un modelo de negocio de un restaurante móvil basado en el método running lean en la cidudad de Chiclayo. Chiclayo.

INCAE. (2018). Lean Canvas: un lienzo para emprendedores (pag. 1). Obtenido de https://www.incae.edu/es/blog/2018/05/21/lean-canvas-un-lienzo-paraemprendedores.html

Lefcovich, M. (2008). Las pequeñas empresas y las causas de sus fracasos. Obtenido de En Madrid. España:

https://degerencia.com/articulo/las_pequenas_empresas_y_las_causas_de_sus_frac asos/

Medina, J. E. (2007). Modelo Integral de Productividad. Bogota: Fondo de Publicaciones Universidad Sergio Arboleda.

Megias, J. (2011). Herramientas: El lienzo de modelos de negocio. . Obtenido de https://javiermegias.com/blog/2011/11/herramientas-el-lienzo-de-modelos-denegocio-business-model-canvas/

Mejía, C. (2003). La Propuesta de valor. Obtenido de http://www.planning.com.co/bd/mercadeo_eficaz/Julio2003.pdf

Mejía, D. (2015). Actitud emprendedora de los egresados de la carrera de administración de empresas de la Universidad Católica Santo Toribio de Mogrovejo. Chiclayo: Universidad Católica Santo Toribio de Mogrovejo .

Mullins, J. \& Komisar, R. (2010). Mejorando el modelo de negocio: cómo transformar su modelo de negocio en un plan $B$ viable. Barcelona: Profit. 
Nestor, A. 2. (2015). Gestión Empresarial y su Influencia en el desarrollo económico de la MYPES del sector servicios, rubro imprentas en la cudad de Tacna, año 2013. Tacna.

Osterwalder, A., \& Pigneur, Y. . (2010). Business model generation (pag.22). New Jersey: Deusto.

Pastor, A. (2014). Propuesta de modelo de negocio para la asociacion del centro de procesamiento pesquero artesanal en el distrito de Santa Rosa - Lambayeque - 2013. . Chiclayo: Universidad Católica Santo Toribio de Mogrovejo.

Porter, M. (1990). The Competitive Advantage of Nations. Free Press, New York, 1990 \& 1998.

Ries, E. (2012). El método Lean Startup. . Barcelona: Deusto.

Rivera, F. y. (2016). Modelo de negocio para iniciativas en CTEI. Una aplicación para la agroindustria piscícola en el Cauca. Cauca.

Rojas, B. (2017). Modelo de negocios Canvas para la empresa multisectorial de Ayash S.A. en la ciudad de Huaraz - 2017. Huaraz: Universidad César Vallejo.

UruguayXXIII. (2014). Sector Olivícola 2014, de Uruguay XXIII, Promoción de inversiones y exportaciones. Obtenido de http://www.uruguayxxi.gub.uy/informacion/wpcontent/uploads/sites/9/2015/05/Informe-Sector-Oliv\%C3\%ADcola-Noviembre2014.pdf

WorkMeter. (03 de Octubre de 2012). El Blog de WorkMeter. Obtenido de https://es.workmeter.com/blog/bid/229017/la-importancia-de-la-productividadempresarial

XXIII, U. (2014). Sector Olivícola 2014, de Uruguay XXIII, Promocin de inversiones y exportaciones. Obtenido de http://www.uruguayxxi.gub.uy/informacion/wpcontent/uploads/sites/9/2015/05/Informe-Sector-Oliv\%C3\%ADcola-Noviembre2014.pdf 